\title{
Clinical- and cost-effectiveness of a technology supported and solution-focused intervention (DIALOG+) in treatment of patients with chronic depression - study protocol for a multi-site, cluster randomised controlled trial [TACK]
}

Philip IJ McNamee ( $\nabla$ philip.mcnamee@nhs.net)

East London NHS Foundation Trust https://orcid.org/0000-0002-6913-5662

Aleksandra Matanov Matanov

Queen Mary University Institute of Population Health Sciences: Wolfson Institute of Population Health

Lauren Jerome

Queen Mary University Institute of Population Health Sciences: Wolfson Institute of Population Health

Neil Walker

Queen Mary University of London - Whitechapel Campus

\section{Sally Kerry}

Queen Mary University Institute of Population Health Sciences: Wolfson Institute of Population Health

Yan Feng

Queen Mary University of London - Whitechapel Campus

Andrew Molodynski

Oxford Health NHS Foundation Trust

Shonagh Scott

Sheffield Health and Social Care NHS Foundation Trust

Latha Guruvaiah

Gloucestershire Health and Care NHS Foundation Trust

Sue Collinson

Homerton University Hospital

Rose McCabe

City University: City University of London

John Geddes

Oxford University: University of Oxford

\section{Stefan Priebe}

Queen Mary University Institute of Population Health Sciences: Wolfson Institute of Population Health

Victoria Bird

Queen Mary University Institute of Population Health Sciences: Wolfson Institute of Population Health 


\section{Research Article}

Keywords: cluster randomised trial, depression, community care, mental health, solution focused

Posted Date: February 21st, 2022

DOI: https://doi.org/10.21203/rs.3.rs-1303555/v1

License: (c) (i) This work is licensed under a Creative Commons Attribution 4.0 International License. Read Full License

Version of Record: A version of this preprint was published at Trials on March 28th, 2022. See the published version at https://doi.org/10.1186/s13063-022-06181-4. 


\section{Title Page}

3 Clinical- and cost-effectiveness of a technology supported and solution-focused

4 intervention (DIALOG+) in treatment of patients with chronic depression - study protocol

5 for a multi-site, cluster randomised controlled trial [TACK]

6

7 Philip McNamee [corresponding author], Unit for Social and Community Psychiatry, Newham Centre

8 for Mental Health, Cherry Tree Way, S13 8SP, philip.mcnamee@nhs.net

10 Aleksandra Matanov, Unit for Social and Community Psychiatry, Newham Centre for Mental Health,

11 Cherry Tree Way, S13 8SP, a.matanov@qmul.ac.uk

13 Lauren Jerome, Unit for Social and Community Psychiatry, Newham Centre for Mental Health,

14 Cherry Tree Way, S13 8SP, I.jerome@qmul.ac.uk

16 Sally Kerry, Pragmatic Clinical Trials Unit, Centre for Evaluation and Methods

17 Queen Mary University, London, Yvonne Carter Building, 58 Turner Street, London, E1 2AB,

18 s.m.kerry@qmul.ac.uk

19

Neil Walker, Pragmatic Clinical Trials Unit, Centre for Evaluation and Methods

21 Queen Mary University, London, Yvonne Carter Building, 58 Turner Street, London, E1 2AB,

22 neil.walker@qmul.ac.uk

24 Yan Feng, Pragmatic Clinical Trials Unit, Centre for Evaluation and Methods

25 Queen Mary University, London, Yvonne Carter Building, 58 Turner Street, London, E1 2AB,

26 yan.feng@qmul.ac.uk 
Andrew Molodynski- Oxford Health NHS Foundation Trust, Research \& Development, Warneford

Shonagh Scott, Sheffield Health and Social Care NHS Foundation Trust, Research \& Development,

Latha Guruvaiah- Gloucestershire Health and Care NHS Foundation Trust, Research \& Development,

Fritchie Centre, Charlton Lane, Cheltenham, GL53 9DZ, latha.guruvaiah@ghc.nhs.uk

Sue Collinson- Homerton University Hospital, Homerton Row, London E9 6SR, sue.collinson@nhs.net

Rose McCabe- Centre for Mental Health Research, City, University of London, Northampton Square,

London, EC1V OHB, rose.mccabe@city.ac.uk

John Geddes, Department of Psychiatry, University of Oxford, Warneford Hospital, Warneford Lane,

Stefan Priebe, Unit for Social and Community Psychiatry, Newham Centre for Mental Health, Cherry

Tree Way, S13 8SP, s.priebe@qmul.ac.uk

Victoria Bird [corresponding author]- Unit for Social and Community Psychiatry, Newham Centre for 
53 Background: Many with an acute depressive disorder go on to develop chronic depression, despite ongoing care. There are few specifically designed interventions to treat chronic depression. DIALOG+, a technology-assisted intervention based on the principles of solution-focused therapy, may be beneficial. It has been shown to be effective as a treatment for patients with psychotic disorders, especially in regards to increasing quality of life. DIALOG+ was designed to be flexibly applied and not diagnosis-specific, aiming to structure communication and generate a personallytailored care plan. This cluster randomised controlled trial (RCT) is part of a programme of research to adapt and test DIALOG+ for patients with chronic depression.

61 Methods: Patients will be eligible for the trial, if they have exhibited symptoms of depression or 62 non-psychotic low mood for at least 2 years, have regular contact with a clinician and have a low 63 subjective quality of life, and moderate depressive symptoms. Clinicians, who routinely see eligible 64 patients, will be recruited from a number of sites across NHS England. Clusters will have between 1 and 6 patients per clinician and will be randomised in a 1:1 ratio to either the intervention (DIALOG+) or active control group (treatment as usual + DIALOG scale). Clinicians in the intervention group are trained and asked to deliver the intervention regularly for 12 months. Active control participants receive treatment as usual and are asked to rate their satisfaction with areas of life and treatment on the DIALOG scale at the end of the clinical session. Approximately 112 clinician clusters will be recruited to reach a total patient sample size of 376 . Clinical and social outcomes including costs are assessed at baseline and 3-, 6- and 12-months post randomisation. The primary outcome will be subjective quality of life at 12 months.

Discussion: This definitive multi-site, cluster RCT aims to evaluate the clinical- and cost-effectiveness of DIALOG+ for people with chronic depression. If shown to be effective for this patient population it could be used to improve outcomes of mental health care on a larger scale, ensuring that patients with complex and co-morbid diagnoses can benefit. 
78 Trial registration: ISRCTN11301686 ${ }_{2}$ registered: $13 . J u n .2019$

79 Key words: cluster randomised trial, depression, community care, mental health, solution focused 80 


\begin{tabular}{|c|c|}
\hline Title $\{1\}$ & $\begin{array}{l}\text { Clinical- and cost-effectiveness of a technology supported and solution- } \\
\text { focused intervention (DIALOG+) in treatment of patients with chronic } \\
\text { depression - study protocol for a multi-site, cluster randomised controlled } \\
\text { trial [TACK] }\end{array}$ \\
\hline $\begin{array}{l}\text { Trial registration }\{2 \mathrm{a} \text { and } \\
2 \mathrm{~b}\} \text {. }\end{array}$ & ISRCTN11301686; https://doi.org/10.1186/ISRCTN11301686 \\
\hline Protocol version $\{3\}$ & Study Protocol v9.0, 30.Sep.2022 \\
\hline Funding $\{4\}$ & $\begin{array}{l}\text { The trial makes up part of a programme grant funded by the National } \\
\text { Institute of Health Research (NIHR), Programme Grants for Applied } \\
\text { Research (PGfAR; RP-PG-0615-200010) }\end{array}$ \\
\hline Author details $\{5 a\}$ & $\begin{array}{l}\text { a. Philip McNamee, Aleksandra Matanov, Lauren Jerome, Stefan } \\
\text { Priebe \& Victoria Bird- Unit for Social and Community Psychiatry, } \\
\text { bueen Mary University London } \\
\text { Queen Mary University of London } \\
\text { c. Andrew Molodynski- Oxford Health NHS Foundation Trust } \\
\text { d. Shonagh Scott- Sheffield Health and Social Care NHS Foundation } \\
\text { e. Latha Guruvaiah- Gloucestershire Health and Care NHS Foundation } \\
\text { f. Sue Collinson- Homerton University Hospital }\end{array}$ \\
\hline
\end{tabular}




\begin{tabular}{|c|c|}
\hline & $\begin{array}{l}\text { g. Rose McCabe- Centre for Mental Health Research, City University of } \\
\text { London } \\
\text { h. John Geddes- Department of Psychiatry, University of Oxford }\end{array}$ \\
\hline $\begin{array}{l}\text { Name and contact } \\
\text { information for the trial } \\
\text { sponsor }\{5 \mathrm{~b}\}\end{array}$ & $\begin{array}{l}\text { Noclor is the sponsor of the trial on behalf of East London NHS Foundation } \\
\text { Trust. } \\
\text { Address: 1st Floor, Bloomsbury Building St Pancras Hospital, 4 St } \\
\text { Pancras Way, London, NW1 OPE } \\
\text { Tel: } 02076855949 \\
\text { E: sponsor.noclor@ nhs.net }\end{array}$ \\
\hline Role of sponsor $\{5 c\}$ & $\begin{array}{l}\text { The sponsor has the responsibility for proportionate and effective } \\
\text { arrangements being in place to set up, run and report the research project } \\
\text { to a high standard that meets the requirements of good clinical practice. }\end{array}$ \\
\hline
\end{tabular}

82 


\section{Introduction}

\section{Background and rationale}

85 The effective treatment of depression is a priority within the NHS [1], not just because of its relatively

86 high prevalence, but also because it is a leading cause of disability worldwide [2]. The economic

87 burden on the NHS and wider society is high, due to patients being high utilisers of healthcare services as well as experiencing work productivity impairments [3]. Despite existing evidence-based

89 interventions aiming to reduce the impact of depression, there has been no reduction in the global

90 prevalence or burden of depression since 1990 [4], and the number of people experiencing depression

91 within the UK is set to increase to 1.45 million by 2026 [5].

92 Furthermore, over a third of people who experience an acute episode of depression do not

93 adequately improve and instead go on to develop a chronic disorder, often labelled 'treatment

94 resistant' [6]. Chronic depression is associated with poor clinical and social outcomes including an

95 increased suicide risk, poor quality of life, physical comorbidity, reduced social networks and

96 functional impairment $[5,6,7]$. Chronic depression is broadly defined as 2 years of continuous

97 symptoms in individuals with mood disorder [8]. Past research has tended to focus on the treatment of episodic depression, resulting in a lack of evidence-based interventions specifically tailored for

99 chronic forms [9]. Chronic depression is linked with worse socioeconomic and interpersonal

100 conditions than episodic depression $[10,11]$ and large numbers of chronically depressed patients do

101 not receive appropriate treatment [12]. Many patients with chronic depression in the UK are

102 managed in secondary mental health services and receive treatment from clinicians from a range of

103 fields (e.g., psychiatrists, mental health nurses, social workers, support workers etc.) known as a care

104 coordinator. Care coordination involves regularly meeting with a named mental health professional

105 to co-ordinate the assessment and planning of their care, including regular reviews. However, these

106 meetings are not founded on evidence-based methods to improve outcomes and vary widely

107 between sites [13]. Furthermore, established pharmacological and psychological treatments, such as 
antidepressants or psychotherapy have at best only limited efficacy for this patient group [14].

109 Consequently, there is a need to develop interventions that are both clinically and cost-effective

110 which can be routinely implemented within different clinical settings to make routine care more

111 effective in improving patient outcomes.

112

113 DIALOG+, a technology-assisted and resource-oriented intervention, represents one possible

114 treatment solution. This intervention structures communication between patients and their clinicians

115 during routine meetings in mental health care settings, aiming to create better treatment plans and

116 improve clinical outcomes. DIALOG + consists of a patient-centred assessment (containing 8 quality of

117 life areas and 3 treatment aspects) whereby patients rate their satisfaction with these 11 different

118 areas of life and treatment, on a tablet computer. These routinely collected scores can then be

119 integrated into the discussion between clinician and patient, and used to compare ratings between

120 different areas in the same session, or across the same area over time. The ratings are also used to

121 select up to 3 of the areas for more detailed discussions. This discussion is guided by a 4-step approach,

122 informed by the principles of brief solution-focused therapy. The effectiveness of DIALOG+ was

123 previously established for patients with psychosis treated in the community [15]. A single site, cluster

124 randomised controlled trial with this population found that patients who used the intervention over

1256 months had improved quality of life, fewer unmet needs, lower general symptom levels, better social

126 outcomes and lower NHS treatment costs [16].

128 Previous research has indicated that patients with chronic depression typically have an even lower 129 quality of life compared to those with psychotic disorders [17], meaning that an intervention like 130 DIALOG+ which targets quality of life has increased scope to improve satisfaction and recovery. There 131 is an emerging evidence base from the application of DIALOG+ in small controlled trials in Lower

132 Middle-Income Countries (LMICs) $[18,19]$ that the intervention is suitable and effective in those with 133 depression. However, patients involved in these trials had less chronic forms of depression, and a 
134 definitive, and amply powered trial is required to test the clinical and cost effectiveness of DIALOG+

135 in improving treatment outcomes of patients with chronic depression.

137 The trial makes up a substantial part of the "Tackling Chronic Depression" (TACK) Programme Grant

138 (RP-PG-0615-20010), the overall aim of which is to adapt DIALOG+ to the needs of patients with

139 chronic depression and test its effectiveness. Following earlier exploratory work where clinicians and

140 patients tested the use of DIALOG+ in routine sessions for a 3-month period, and were then

141 interviewed about their experiences, found the basics of the intervention needed no fundamental

142 changes to make it appropriate for this specific patient population [20]. This was followed by a multi-

143 site feasibility randomised controlled trial (in prep) which demonstrated that the intervention was

144 acceptable and feasible and that the trial procedures were appropriate.

\section{Objectives}

147 The primary objective of this definitive trial is to establish whether the regular use of DIALOG+ over a

148 12-month period, in various clinical settings, can improve quality of life in patients with chronic 149 depression, compared with an active control.

150 Secondary objectives are:

151 - To evaluate whether the intervention improves secondary outcomes such as depression

152 symptom severity, treatment satisfaction, and health-related quality of life.

153 - To assess the costs of intervention delivery and to establish the cost-effectiveness of the

154 intervention.

155 - To explore the implementation of the intervention, particularly in regards to clinician training requirements and fidelity to the manual. 
159 A pragmatic cluster-randomised controlled trial design will be used to test the study objectives.

160 Clinicians, and their patients (who together form a cluster), will be randomly allocated to either the experimental (DIALOG+) group or to an active control group (Treatment As Usual (TAU) + DIALOG scale). Clinicians will act as the unit of randomisation, with clustering by clinician to prevent contamination effects within the study. Clinicians allocated to the experimental arm will use DIALOG+ to structure their routine sessions over a 12-month period. Clinicians allocated to the active control arm will deliver routine care but additionally ask the patient to complete the 11-item DIALOG scale on a tablet computer at the end of every session, but without any clinical input or

\section{7 discussion of the items.} In both arms the interventions will be delivered within the context of routine care and therefore will be delivered wherever or however these routine meetings usually take place. This could be within community mental health services, outpatient clinics, GP surgeries and/or at the patient's home, or delivered remotely over the phone or on NHS Trust-approved web-conferencing platforms (e.g., MS Teams). No additional sessions or clinician time will be required to delivered the intervention.

173 Clinicians will be recruited first, and will then identify eligible patients from their caseloads. Cluster sizes will range from a minimum of 1 to a maximum of 6 patients, with an average cluster size between 3 and 4. Randomisation will take place once the cluster is complete- either when the maximum cluster size is reached or when no more eligible participants can be identified from the

177 clinician's caseload.

179 Clinicians will use the intervention, with each patient, monthly (on average) for the first 6 months

180 with additional sessions during the following 6 months (e.g., at 8 and 10 months) at the clinician's discretion. 
183 There will be four data collection points: baseline, 3-, 6- and 12-months after the date of 184 randomisation (see Table 2).

185 An internal pilot was conducted within three of the trial sites (Oxford Health NHS Foundation Trust,

186 Gloucestershire Health \& Social Care NHS Foundation Trust and Sheffield Health and Social Care NHS

187 Foundation Trust) during the first four-months of the trial. Stop-Go criteria were developed a priori

188 based on recruitment rates and clinician training rates. The trial launched on the $26^{\text {th }}$ June 2019 , and

189 criteria for continuation of the trial were met according to the Programme Steering Committee.

190 Data collected as part of the internal pilot will be analysed alongside all other trial data.

191 The SPIRIT Reporting guidelines [21] were used to structure this protocol. The completed SPIRIT

192 figure can be found at Table 2. The full SPIRIT checklist can be found as an additional file.

193 Methods: Participants, interventions and outcomes

194 Study setting

195 This multi-centre study will be coordinated by the East London NHS Foundation Trust (ELFT), based

196 at the Newham Centre for Mental Health. Trial sites, all of which will be NHS England mental health

197 trusts, will be purposefully selected based on eligible patient numbers and to represent a mix of urban,

198 semi-urban and rural areas to allow for variation in demographics amongst the sample (a list of current

199 sites can be seen in Table 1). Multi-disciplinary staff from community mental health teams (CMHTs),

200 including Older Adult services, as well as intermediary and primary care services (where available

201 within secondary care Trusts), will be approached for inclusion in the study.

202 The study was designed with complexity and diversity in mind, given both the variability of care

203 coordination practices across the UK [13] and the differing definitions of chronic depression [7]. The

204 study team adopted an inclusive approach in the design of the trial, particularly in the eligibility 
criteria, to ensure the trial was pragmatic as possible and reflected clinical reality in the treatment of chronic depression with the UK.

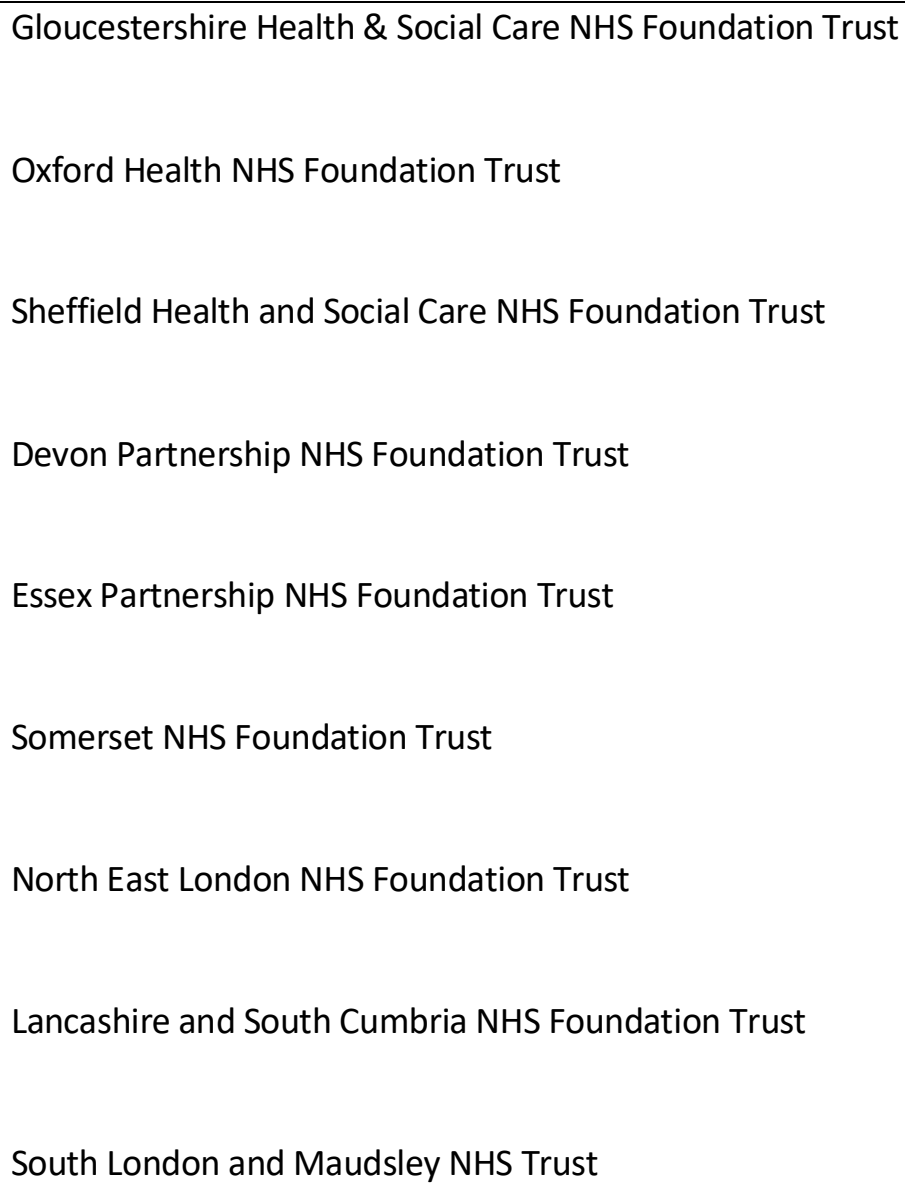

\section{Eligibility Criteria}

210 To reflect the pragmatic nature of the trial, there are broad and inclusive inclusion criteria for both

211 clinicians and patients.

\section{$213 \quad$ Clinicians}

214 Eligible clinicians are any person working as a mental health or healthcare professional within the

215 selected NHS Trust sites (e.g., support workers, mental health nurses, occupational therapists,

216 psychiatrists etc); have at least 6 months experience of working in a healthcare setting; regularly see 
217 their patients on at least a monthly basis; have experience of treating those with chronic depression

218 and have no plans to leave their post within the next 6 months.

219

220 Clinicians are excluded if they have previous experience of using DIALOG+ or if they cannot identify at least 4 eligible patients on their caseload at the time of consenting to the study.

\section{Patients}

224 Patients are eligible if they are between 18 and 100 years old; are currently exhibiting symptoms of depression or non-psychotic low mood with a duration of illness of at least 2 years; are currently receiving treatment from an NHS mental health service with regular contact with the same clinician; have the capacity to provide informed consent and have the ability to speak and understand English to such a degree they can engage with DIALOG+ and complete the research assessment. Following findings from the feasibility trial that chronic depression is often poorly indexed on clinical systems, the inclusion criteria was purposefully based on clinical presentation of chronic depression symptoms as opposed to a diagnosis of chronic depression disorder (e.g. F33 or F34 on the ICD-10). The treating clinician will act as the patient identifier and will use their professional judgement and access to the patient's medical records to decide if symptoms indicating chronic depression are present. Patients with co-morbid diagnoses such as anxiety disorders and/or emotionally unstable personality disorder are eligible for inclusion. both a low quality of life and adequate evidence of current depression symptoms to be eligible.

239 Patients must have a score of less than 5 on the Manchester Short Assessment of Quality of Life 240 (MANSA) [22] and a score of 10 or more on the Montgomery-Åsberg Depression Rating Scale 241 (MADRS) [23]. 
243 Patients will be excluded if they have a primary diagnosis of a substance misuse problem; a diagnosis

244 of an organic mental disorder (F00-F09); are an inpatient on a psychiatric ward at the time of

245 recruitment or do not have regular clinical contact with a mental health professional.

247 Informed consent

248 Informed consent will be obtained by trained researchers either in person or remotely from the

249 individual participant, with a signed copy of the form being made available to the participant.

250 At the point of consent, patients will have the option to agree to one of their sessions being recorded

251 (either video or audio recorded) in order to assess adherence to the intervention, and for patients to

252 be invited to a one-to-one interview at the end of their intervention period to discuss their 253 experiences.

254 All data will be held on NHS password-protected computers or stored on NHS premises to maintain 255 confidentiality.

\section{Interventions}

257 Both the experimental intervention (DIALOG + ) and the active control intervention (TAU + DIALOG

258 Scale) will be used as part of routine care; therefore participants will continue to receive all standard

259 treatments as part of their care. This includes medication, referral to other psychological

260 interventions and social prescribing interventions. There are no contraindications for any other

261 treatment and care should continue for all participants as normal. DIALOG+ and the DIALOG scale

262 are supported by an iOS app (DIALOG v1.9.0), which will be preloaded on to an Apple iPad tablet,

263 and provided by the research team prior to the clinician training.

264 The frequency of sessions will replicate what is standard for that clinician-patient dyad, although

265 clinicians are required to deliver the interventions at least once a month for the first 6 months of the

266 intervention delivery period. 
DIALOG+ provides an evidence-based structure to routine clinical appointments between clinician and patient. Clinicians will therefore be instructed to conduct their routine care coordination sessions, planned with their consented patients, using the steps, scales and structure offered by the intervention. DIALOG+ consists of two main parts: 1) a patient-centred assessment whereby the clinician invites the patient to rate their satisfaction with different life domains and treatment aspects (the DIALOG scale), followed by 2) a four-step approach based on the principles of solution-focused

274 therapy.

The DIALOG scale is a computer-mediated procedure to rate 11 areas of life. Patients are asked to rate their satisfaction with eight areas of life (mental health, physical health, job situation, accommodation, leisure activities, relationship with family/partner, friendships, personal safety) and three treatment areas (medication, practical help, and meetings with mental health professionals). Each satisfaction item is rated on a rating scale of $1-7$, from 'Totally Dissatisfied' to 'Totally Satisfied'. The 11 areas are presented in a fixed order, and following each question, the patient is asked to rate whether they would like more help within each area [24]. Following review of the scores across the 11 areas, which includes comparing the current ratings with the ratings obtained from any previous session, up to three of the areas that are listed on the DIALOG scale are chosen to be discussed in more detail. The four-step solution-focused approach is used to structure the discussion so as to identify patients' resources and develop solutions to deal with the patients concerns. At all times the ratings on the scale are referred to in order to underpin and contextualise the discussion. Step 1, Understanding, elicits contextual information about the area under discussion and establishes what is working in that area. Step 2, Looking Forward, asks the patient to adopt a future perspective and think about the 'best case scenario' within that domain as well as the smallest improvement that can be made to incrementally move up the rating scale. Next, 
292 Step 3, Considering Options, invites the patient to reflect on what they and others can do to in order

293 to improve quality of life. Finally, Step 4, Agreeing on Actions, summarises the discussion and a list of

294 actions are created and inputted into the system. Ultimately the clinician and patient together will create an action plan, made up of individual action items for each of the discussed areas to be completed before the next session.

298 For a more detailed description of DIALOG+ please see [16] and the DIALOG+ website [25].

299 All clinicians allocated to the intervention arm will receive the standardised DIALOG+ training which

300 was developed earlier on in the programme of research. Standardised training comprises of a one-off 301 session of $60-90$ minutes. This is followed by a mandatory "top-up" session once delivery of the 302 intervention by the clinician has begun. For practical reasons the training will most frequently be 303 carried out one to one, although where timings and practicalities allow, group training sessions will be 304 allowed. Training will be facilitated by a trained researcher or the trial manager.

305 Training will take place as soon as possible after randomisation and can take place either face-to-face or via a Trust approved web conferencing platform. A "train the trainer" model has been created

307 whereby a senior member of the core research team can train other unblinded researchers to conduct

308 training with clinicians. During the training session, clinicians are taught about the developmental 309 history of the intervention, given a practical demonstration of how to use and navigate the app using 310 the tablet computer, informed about the evidence for its effectiveness, and shown patient and 311 clinician testimonials of those who have experience of using it. Clinicians will also be shown training 312 videos (commissioned by the research team), and have the opportunity to participate in a role play 313 exercise. Clinicians will also be provided with the DIALOG+ manual and further reading. 
314 Throughout the duration of the study clinicians can contact the trainers for support at any time. In

315 addition, clinicians will also be offered at least one hour of clinical supervision. This supervision will be

316 project-specific (i.e., additional to routine supervision) and provided by a trained therapist.

317 Clinicians (or patients) in the intervention arm may decide to continue with DIALOG+ after the end of

318 the main intervention period (i.e., the first 6 months of delivery). This will be documented and

319 considered in the analysis of outcomes after the follow-up period.

320 Active Control Arm (DIALOG scale + TAU)

321 The active control condition includes treatment as usual plus a defined intervention that also involves

322 the use of a tablet and an assessment of the patient's quality of life. At the end of every routine

323 session, clinicians in the control condition, will hand the iPad to the patient and ask them to rate their

324 satisfaction on the 11 areas of the DIALOG scale. The ratings should be completed after every routine

325 meeting, to control for novelty effects (i.e., presence of a tablet) and repeated quality of life

326 assessments. Patients will complete the scale alone without any input or further discussion from the

327 clinician.

328 Clinicians allocated to this group will receive a shorter training session of around 15 minutes, to

329 introduce them to the DIALOG app and explain how they should collect the scale ratings after each

330 routine session.

331 Provisions for post-trial care

332 All participants at the point of finishing participation in the trial will be offered a "mental health

333 resources list" which features contact details of local organisations who offer support. Researchers

334 will also offer all participants a "welfare call" one week after the completion of the 12-month follow 335 up. 
337 The trial will collect information on a range of health, social and cost-related outcomes. The scalebased measures are all well established and have been validated for use with patients with depression. All measures used in the main trial were found to have acceptable completion rates in the feasibility trial.

342 Outcome measures will be completed on a standardised Case Report Form (CRF) at baseline, at the end of the first six-month intervention block (6-month follow up) and at the end of the intervention period (12-month follow up). A shorter assessment, containing only two outcome measures (MANSA [22] \& the Beck Depression Inventory (BDI-II; [26]) will also be collected at 3 months for purposes of imputation.

The primary outcome is subjective quality of life, measured on the MANSA [22].

349 Secondary outcomes for the trial are:

- Depression symptoms as measured via observer ratings on the Montgomery-Åsberg Depression Rating Scale (MADRS) [23] and self-reported on the BDI-II [26]. Scale [28]. This is clinician-rated by the patient's clinician.

- Capability of the general adult population measured on the ICECAP-A [29].

- Health related quality of life measured by the EQ-5D-5L instrument (EQ-5D-5L) [30].

- Costs of health service use, prescribed medication, productivity lost, burden on family and friends, and contact with criminal justice, assessed on the Client Service Receipt Inventory (CSRI [31])

- Costs of treatments from both trial arms, and costs of supervision and training to clinicians, assessed on Health Economics Inventory Forms developed by the trial health economists. 
- Additionally, there will be a 'DIALOG+ Experience Questionnaire' completed at 6- and 12months by those patients allocated to the intervention arm. This is a purposefully developed measure by the trial team in collaboration with the Lived Experience Advisory Panel (LEAP) to investigate the patient experience of receiving DIALOG+ as part of routine care.

367 Table 2. SPIRT figure outlining schedule of enrolment, interventions, and assessments

\begin{tabular}{|c|c|c|c|c|c|c|}
\hline & \multicolumn{6}{|c|}{ STUDY PERIOD } \\
\hline & Enrolment & Allocation & & Post- & location & \\
\hline TIMEPOINT** & $-t_{1}$ & 0 & $\begin{array}{c}t_{1} \\
\text { (Baseline) }\end{array}$ & $\begin{array}{c}t_{2} \\
\text { (3 months) }\end{array}$ & $\begin{array}{c}t_{3} \\
\text { (6 months) }\end{array}$ & $\begin{array}{c}t_{4} \\
\text { (12 months) }\end{array}$ \\
\hline \multicolumn{7}{|l|}{ ENROLMENT: } \\
\hline Eligibility screen & $x$ & & & & & \\
\hline \multirow{3}{*}{$\begin{array}{r}\text { Informed consent } \\
\text { Manchester Short } \\
\text { Assessment of } \\
\text { Quality of Life } \\
\text { (MANSA) } \\
\text { Montgomery- } \\
\text { Åsberg Depression } \\
\text { Rating Scale } \\
\text { (MADRS) }\end{array}$} & $x$ & & & & & \\
\hline & $\mathrm{x}$ & & $\mathrm{x}$ & $x$ & $\mathrm{x}$ & $x$ \\
\hline & $x$ & & $x$ & & $\mathrm{x}$ & $x$ \\
\hline Allocation & & $x$ & & & & \\
\hline \multicolumn{7}{|l|}{ INTERVENTIONS: } \\
\hline \multicolumn{7}{|l|}{ DIALOG+ } \\
\hline \multicolumn{7}{|l|}{ TAU + DIALOG Scale } \\
\hline \multirow{3}{*}{$\begin{array}{l}\qquad \text { ASSESSMENTS: } \\
\text { Beck Depression } \\
\text { Inventory (BDI-II) } \\
\text { Clinical Global } \\
\text { Impression (CGI) }\end{array}$} & & & & & & \\
\hline & & & $x$ & $\mathrm{x}$ & $\mathrm{x}$ & $\mathrm{x}$ \\
\hline & & & $\mathrm{X}$ & & $\mathrm{x}$ & $x$ \\
\hline
\end{tabular}




\begin{tabular}{|c|c|c|c|}
\hline \multirow{2}{*}{$\begin{array}{l}\text { Scale: Severity of } \\
\text { Illness subscale } \\
\text { ICECAP-A }\end{array}$} & & & \\
\hline & $x$ & $X$ & $X$ \\
\hline $\begin{array}{l}\text { Client Satisfaction } \\
\text { Questionnaire } \\
\text { (CSQ-8) }\end{array}$ & $x$ & $x$ & $X$ \\
\hline $\begin{array}{l}\text { Euroqol } 5 \\
\text { dimension (EQ-5D- } \\
5 L \text { ) }\end{array}$ & $X$ & $x$ & $x$ \\
\hline $\begin{array}{l}\text { Client Service } \\
\text { Receipt Inventory } \\
\text { (CSRI) }\end{array}$ & $X$ & $X$ & $x$ \\
\hline \multicolumn{4}{|l|}{$\begin{array}{l}\text { Training, } \\
\text { supervision and } \\
\text { treatment costs }\end{array}$} \\
\hline $\begin{array}{l}\text { DIALOG+ } \\
\text { Experience } \\
\text { Questionnaire }\end{array}$ & & $\begin{array}{c}X \\
\text { (Intervention } \\
\text { arm only) }\end{array}$ & $\begin{array}{c}\mathrm{X} \\
\begin{array}{c}\text { (Intervention arm } \\
\text { only) }\end{array}\end{array}$ \\
\hline
\end{tabular}

369 All assessments are conducted by a trained researcher on NHS premises, in the community or

370 remotely. Researchers assessing the outcomes are blinded to the allocation of the patient.

371 The list of outcome measures was decided upon through consultation with the Programme Steering

372 Committee, the LEAP and the Pragmatic Clinical Trials Unit (PCTU).

374 Patient participants will be paid a $£ 20$ voucher for their time when completing assessments at 6 - and

375 12-month follow ups.

376 For clinicians, sociodemographic and information about their professional background including time

377 spent working in mental health services will be collected via questionnaires at the point of

378 recruitment.

379 Data collected on paper CRFs will be entered into the online OpenClinica database by trained, blinded

380 researchers. There will be regular data monitoring visits organised by the PCTU where prime source

381 data and data entry into the database will be reviewed. 
383 The original sample size calculation was based on data from the previous DIALOG+ trial [15]. A standardised effect size of 0.35 on the MANSA (representing a mean difference of $0.31(S D=0.9)$ ) is equivalent to an improvement in satisfaction ratings of at least one point (on a 7-point scale) on four out of the 11 life and treatment areas on the DIALOG scale. The effect size was chosen as such an improvement is regarded as clinically meaningful [15], and related to noticeable improvement in subjective quality of life.

To detect an effect size of 0.35 (SD = 1) on the MANSA scale, and setting power at $90 \%$ for $5 \%$ significance, the total number of patients required was 172 per group $(n=344)$. After accounting for clustering based on an ICC of 0.01 (as observed within the original DIALOG+ trial relating to subjective quality of life (SQoL) as measured by the MANSA), a conservative design effect of 1.04 and allowing for a drop-out rate of $20 \%$, a total of 448 patients were needed to be recruited to give an analysable sample of 358 (179 per group). Therefore 112 clinicians were needed to be recruited, with an average of four patients per cluster. The numbers of potentially eligible patients per clinician varies by site and team, so an estimate of four patients per clinician was used in the feasibility trial and this was found to be achievable. Cluster sizes in the feasibility trial ranged from $3-5$.

Following analysis of the TACK feasibility trial data (in prep), the power calculation was revised,

399 integrating the correlation coefficient between at baseline and final follow up, on the primary 400 outcome (MANSA). The lower end of the $95 \%$ confidence interval for the correlation coefficient was 401 used (0.4). All other assumptions remained the same as for the original power calculation i.e., an effect 402 size of $0.35(S D=1)$, power set at $90 \%$ and a design effect of 1.04 . The updated power calculation gave 403 a target sample size of 376 , with a projected analysable sample of 300 (150 per group) when a $20 \%$ 404 drop out rate was accounted for. 
406 As a multi-site trial, participants will be recruited from nine NHS sites and a number of clinical settings.

407 As reflected in the inclusion criteria, any clinical team commissioned by the secondary mental health

408 Trusts will be eligible for inclusion, so long as they meet the requirements of the session frequency

409 and length dictated by DIALOG+ (this includes intermediary or primary care services where there is

410 integration and a clear link to the secondary care Trust, including Improving Access to Psychological

411 Therapies (IAPT) services).

412 Researchers will actively identify eligible clinical teams and individual staff members. Researchers will

413 attend weekly multi-disciplinary team meetings to present the research study with potentially eligible

414 clinicians/ teams. Clinical teams who work with non-psychotic patients will be specifically targeted, to

415 increase the possibility of eligibility.

416 After recruiting eligible clinicians, the caseload of each clinician will be screened and eligible patients

417 identified. Members of the clinical team will approach patients and gain assent for contact by the

418 research team. A local researcher will determine eligibility and obtain informed consent followed by

419 completion of the screening measures and the remainder of the baseline Case Report Form (CRF),

420 where the patient is eligible.

422 Randomisation will be carried out remotely via e-mail from the trial manager to an independent

423 statistician at the PCTU, Queen Mary University of London. The unit of randomisation is the clinician

424 with an allocation ratio of 1:1. Randomisation will be stratified by site in blocks of 4 , ensuring balanced

425 numbers of patients in each trial arm at each NHS Trust. The allocation sequence will be via site lists

426 created by the independent statistician on a protected server. The resulting allocation will be emailed

427 back to the trial manager who will then inform the unblinded researcher at the relevant site. The local

428 unblinded researcher will then be responsible for informing the clinician of their allocation. 
430 The trial manager will be unblinded to all allocations and take overall responsibility for overseeing the

431 randomisation process. Only the trial manager can request clusters for randomisation after liaising 432 with the independent statistician to ensure that randomisations are recorded and the correct people 433 informed.

434 Each trial site will have at least one blinded and one unblinded researcher, therefore allowing the 435 unblinded researcher to be aware of the allocation of each cluster and to inform the clinician and 436 arrange training etc. All other study staff, including the principal investigators, will remain blinded.

437 Due to the nature of the intervention, clinicians and patients will be aware of their allocation.

438 Patient participants will be asked to not discuss the treatment they received with researchers at the 439 data collection time points to avoid unblinding research staff.

440 If a researcher is unblinded accidently, or where the unblinding of a researcher is required (i.e., a 441 principal investigator being required to assess the seriousness of a related serious adverse event) then 442 a note will be made on the local system so that those participants will have no further direct contact 443 with those that have been unblinded. Where blinding cannot be maintained or is broken, researchers 444 from the coordinating centre will be used to help support local sites and provide research capacity.

Statistical Methods

447 The primary outcome analysis of quality of life, as measured on the MANSA, will be conducted using

448 a mixed effects model to adjust for clustering and including baseline level of the MANSA and NHS

449 site as covariates, as well as key demographic variables, (that are known to affect outcome) and

450 illness severity. The treating clinician will be fitted as a random intercept effect. 
451 The analysis will use intention-to-treat analysis by including all patients in the arm to which they were

452 randomised, whether or not they received the intervention and including all patients in the analysis

453 by using multiple imputation where outcomes are missing. Results will be presented as an adjusted

454 mean difference.

455 Each secondary outcome will be analysed using a mixed effects model to allow for clustering and

456 adjusting for NHS site and baseline value of the outcome.

457 The statistical team will remain blinded to the allocation of clusters until the database is finalised and

458 locked for analysis.

459 Subgroup analyses may be conducted post-hoc as a result of the variance in intervention delivery

460 caused by the COVID-19 pandemic (see 'COVID-19 Amendments' section).

461 A full statistical analysis plan will be written before data collection is complete, signed off by the Programme Steering Committee (PSC), and will be available via the project website.

\section{Health economic evaluation}

464 In the economic evaluation alongside the trial, we will measure the generic health related quality of

465 life of participants together with the costs of providing DIALOG+ and TAU, other health/social care

466 and societal costs of participants over a 12-month period. We will assess the cost-effectiveness of

467 DIALOG+ from NHS and personal social services perspectives following the intention-to-treat principle.

468 The resource usage data for delivering interventions and training/supervising clinicians will be collected by TACK researchers using purposefully developed health economics inventory forms. Other resource usage data will be collected from patients using a customised interview-based CSRI at

471 baseline, 6-month and 12 months follow ups. Costs for each resource item will be calculated as a 
472 product of the quantity of resource used and its corresponding unit cost. Cost items will be summed

473 together and presented at patient and assessment point level.

474 The primary outcome for economic evaluation will be EQ-5D-5L index scores, converted to quality 475 adjusted life years (QALYs) using the UK EQ-5D-5L value set [30]. We will conduct descriptive analyses 476 to compare the costs and outcomes between the two trial arms at each assessment point.

477 Cost-effectiveness analyses will evaluate differences between patient's total costs and QALYs between 478 trial arms. We will use a multiple Imputation approach to handle missing data. An incremental cost479 effectiveness ratio (ICER) will be calculated as the extra costs incurred to produce an extra QALY. The 480 ICER will be compared to the thresholds for cost-effectiveness typically used by NICE in the UK, i.e., $481 £ 20,000$ to $£ 30,000$ [33]. Uncertainty around the estimated ICER will be presented by the cost482 effectiveness plane [34] and cost-effectiveness acceptability curve [32].

483 In the sensitivity analysis, we will (1) conduct cost-effectiveness analyses under alternative scenarios related to implementation (e.g., different combination of staff) to help contextualise the findings for future implementation (2) use a wider perspective by including costs from productivity lost, family and friends support, and contact with criminal justice services (3) analyse the data for a scenario using

487 ICECAP-A as an alternative QALY outcome measure [29, 34]. Finally, if the intervention demonstrates 488 effectiveness during the 12-month trial period, we will study its longer-term cost-effectiveness over 489 24-month period after the baseline point.

490 A full health economic analysis plan will be written before data collection is complete, and will be 491 available via the project website. 
493 No formal interim efficacy analyses have been planned for the trial data. Data completeness of

494 outcome measures will be assessed and presented to the Data Monitoring and Ethics Committee

495 (DMEC) every 6 months during the trial.

497 In parallel with the trial, an embedded process evaluation will complement the results of the cluster

$498 \mathrm{RCT}$, and will use three different sources of data to enhance the understanding of how DIALOG+ is

499 delivered, the mechanisms of change, and identify the possible barriers to wider implementation.

500 1) In-depth interviews will be conducted post-intervention with approximately 36 patients and 24

501 clinicians purposively sampled; 2) video and audio recordings will be taken of a sample of DIALOG+

502 sessions and adherence to the intervention manual will assess fidelity; 3 ) routinely collected data from

503 the DIALOG app will be extracted from the clinician's iPad at 12 month's post-randomisation which

504 will give data about quality of life rating changes as well as insight into the number of sessions, length

505 of sessions, what items were selected as needing more help, and which items were selected for further

506 discussion etc.

Process Evaluation Analysis

508

509 Patients and clinicians who agree to a post-intervention interview will have sound files transcribed

510 and analysed using framework analysis, with analysts looking for data pertaining to the experience

511 of receiving/ delivering the study intervention.

512

513 Video and audio recordings of DIALOG+ sessions will be analysed using the DIALOG Adherence Scale

514 (v2), to check for fidelity to the core components of the DIALOG manual and training. This will help

515 identify key areas that are overlooked in the delivery of DIALOG+ and help to improve the training

516 resources. 
518 Routinely collected data will be extracted from the clinician iPads and entered onto a database

519 where descriptive data will be presented in relation to number of sessions, length of sessions, SQoL

520 ratings (and their variance over time), and action items set.

\section{$521 \quad$ Oversight \& Monitoring}

522 Both a PSC and a Data Monitoring and Ethics Committee (DMEC) have been convened to provide

523 oversight to the trial. The PSC is chaired by an independent academic clinician and the DMEC chaired

524 by an independent statistician. Both committees meet at least every 6 months to review project

525 progress.

526 Adverse event reporting

527 Any serious adverse events will be recorded in a specific CRF form and their relatedness to the DIALOG+ intervention will be adjudicated by the principal investigator from the local site. All principal investigators are senior clinicians.

530

531 SAEs that are unexpected or related to the intervention will be reported to the study sponsor. Upon

532 the event being resolved the data from the CRF will be entered onto the trial online database for

533 reporting purposes.

\section{Dissemination Plans}

535 Throughout all phases of the programme of research, the study team will disseminate information

536 about the activities and results of the trial through social media and a project specific website [36] in

537 order to reach a wider public audience. When results become available, they will be disseminated

538 through:

539 
- Scientific publications in peer-reviewed open-access journals.

- Presentations at national and international conferences and to professional and nonprofessional audiences at appropriate events.

- Existing research and clinical networks, including but not limited to the World Health Organisation (WHO), the NIHR, the Local Clinical Research Network, organisations involved in Quality Improvement initiatives and professional networks of the programme co-

547

Ethics Approval

The study has been approved by the NHS Wales Research Ethics Committee 6 (REC reference

550 19/WA/0160).

Public \& Patient Involvement

552

553 A Lived Experience Advisory Panel (LEAP) has worked in collaboration with the study team over the

554 entire programme of research, including the trial. The LEAP meets regularly to receive updates on

555 study progress and to ensure that study procedures are safe and appropriate for patient

556 participants. The LEAP reviewed all patient facing trial documents, and developed the DIALOG+

557 Experience Questionnaire (a bespoke measure used as part of the trial). The LEAP will play an active 558 role in the dissemination of the trial findings.

559

Impact of COVID-19 and related amendments to study protocol

561

562 As a result of the COVID-19 pandemic and the sequence of national and local lockdowns during 2020

563 and 2021, a number of amendments were made to the study protocol, these are outlined below. 
565 All research recruitment and randomisation activities were suspended by the study Sponsor from 18

566 March 2020 to 1 September 2020. During this period, delivery of the intervention was allowed to

567 continue (as the intervention replaced routine care) but only for those patient participants already

568 randomised. In addition, the treatment had to be completed remotely. Clinicians were therefore

569 offered additional support and guidance on how to use DIALOG+ (particularly the app) when

570 working remotely. DIALOG + was designed to be an interactive, face-to-face intervention, making use

571 of shared visual references and the collaborative sharing of equipment. Although the delivery of

572 DIALOG+ remotely was sub-optimal- comparative to what was originally envisaged-it was decided

573 by the clinical leads that the potential harm of stopping delivery abruptly was a higher risk than that

574 of delivering DIALOG + in this way. Many aspects of the intervention could continue, such as the

575 rating of the DIALOG scale, the focus on structuring of sessions using the principles of solution

576 focused therapy and setting personalised action items aiming to improve satisfaction.

577

578 Originally all consent obtaining and data collection procedures (at all timepoints) were due to be

579 conducted face-to-face by a trained researcher. In response to social distancing policies and the need for many researchers to work from home, permissions were gained for consent and study data to be collected remotely. Standard operating procedures were developed in collaboration with the study sponsor and the PCTU to ensure that this was completed in a safe and ethical manner. All safeguarding procedures were developed in collaboration with LEAP to ensure that remote collection of sensitive data was not harmful to patient participants and the team implemented strategies such as welfare checks one week after data collection, and localised mental health resources lists to help support patients.

In parallel to the suspension of research activities, many mental health teams, especially those

589 working in the community, were required to stop seeing patients face-to-face, either in clinic or through home visits. In the first UK lockdown from March to June 2020, many mental health services 
591 were restructured, staff seconded, or recovery teams disbanded completely. This led to high levels

592 of dropout of recruited clinicians, and wide-ranging discharges of patient participants from services

593 meaning they could no longer continue on in the trial.

594

Following guidance from the NIHR, the trial was able to restart in September 2020. However, recruitment and data collection procedures continued to be conducted remotely for the full recruitment period duration which led to long lasting disruption to recruitment and follow up rates. extended to 30 months.

600

601 To adjust for any COVID-19 pandemic effects on the intervention itself, the outcomes or both, a

602 sensitivity analysis may be conducted as part of the statistical analysis that will adopt a mixed-effects

603 model approach, grouping different delivery formats of the intervention (i.e., face to face vs remote 604 delivery vs a mixture of both).

605

606

\section{Discussion}

607 At present, large numbers of patients with chronic depression regularly meet clinicians in secondary mental health settings, but these sessions are not guided by evidence-based principles. DIALOG+ is the only intervention specifically developed to make routine patient-clinician meetings in mental

610 health care therapeutically effective. Early evidence from global work [18] has shown promising

611 results for DIALOG + when applied to episodic depression, but a definitive trial is required to see if a

612 generic tool like DIALOG+ can be used on complex and long-term depression.

613

614 DIALOG+ does not require the creation of new specialist services or the restructuring of

615 organisations, but rather can take the time and talent of existing staff to benefit the thousands of 616 patients with chronic depression who require tailored and evidence-based support. Through the 
617 structuring of routine sessions, following the DIALOG+ manualised framework for people with

618 chronic depression, this intervention may be a cost-saving and easily implemented way of improving

619 quality of life, and other clinical outcomes, for this patient group.

620

621 The procedure of DIALOG+ also provides regular and consistent outcome data, i.e., patient ratings of

622 satisfaction with life and treatment. This data cannot only be used to evaluate services on a local,

623 regional and national level, but due to the timing of the trial can also provide an insight into how

624 individual and group SQoL scores were impacted by the COVID-19 pandemic and the subsequent

625 public health measures, which have been shown to have a major effect on mental health,

626 particularly depression [2].

627

628 DIALOG+ is an existing generic and widely applicable intervention, which has been shown to be

629 effective and implementable in a number of different clinical settings and countries. If this definitive

630 trial shows DIALOG+ to be effective in improving outcomes for people with chronic depression then

631 it can strengthen the implementation work already happening both nationally and globally, ensuring

632 that patients with complex, co-morbid and chronic mental health problems can benefit from

633 DIALOG+.

634

635

Trial Status

636 The trial is currently actively recruiting. Recruitment began on the $24^{\text {th }}$ June 2019 and is expected to

637 end by the $30^{\text {th }}$ January 2022. The latest version of the trial protocol is v9.0, 30.Sep.2021 (available

638 from the corresponding author on request).

639

640 Abbreviations

BDI

Beck Depression Inventory

COVID-19 Coronavirus disease 2019 
CMHT Community Mental Health Team

$\begin{array}{ll}\text { CRF } & \text { Case Report Form } \\ \text { CSRI } & \begin{array}{l}\text { Client Services Receipt Inventory } \\ \text { Data Monitoring and Ethics Committee } \\ \text { DMEC }\end{array} \\ \text { ELFT } & \text { East London NHS Foundation Trust } \\ \text { IAPT } & \begin{array}{l}\text { Improving Access to Psychological Therapies } \\ \text { Incremental cost-effectiveness ratio }\end{array} \\ \text { ICER } & \text { Lower Middle-Income Countries } \\ \text { LMICs } & \text { Montgomery-Åsberg Depression Rating Scale } \\ \text { MADRS } & \text { (MADRS) } \\ \text { MANSA } & \text { Manchester Short Assessment of Quality of } \\ & \text { Life } \\ \text { NHS } & \text { National Health Service } \\ \text { PCTU } & \text { Pragmatic Clinical Trials Unit } \\ \text { QALYS } & \text { Quality Adjusted Life Years } \\ \text { RCT } & \text { Randomised Controlled Trial } \\ \text { SQoL } & \text { Subjective Quality of Life } \\ \text { TAU } & \text { Treatment as Usual } \\ \text { WHO } & \text { World Health Organisation }\end{array}$

641

642 Declarations

643 Ethics approval and consent to participate

644 This trial was reviewed and approved by the NHS Wales Research Ethics Committee 6 (19/WA/0160).

645 Written, informed consent to participate will be obtained from all participants.

646

647 Consent for publication

648 A template consent form for both clinicians and patient participants is available on request.

649

$650 \quad$ Availability of data and materials

651 The final trial dataset will be available upon request to the corresponding author once all analysis is

652 complete.

653

$654 \quad$ Competing interests

655 The authors declare that they have no competing interests. 
$657 \quad$ Funding

658 This project is funded by the National Institute for Health Research (NIHR) [Programme Grant for 659 Applied Research (TACK: RP-PG-0615-200010)]. The views expressed are those of the author(s) and 660 not necessarily those of the NIHR or the Department of Health and Social Care.

661

662 Author Contributions

663 VB is the programme co-chief investigator, conceived the study and led on the study protocol

664 development. SP led the intervention development and is the programme co-chief investigator. SK

665 and NW led on the statistical analysis plans. YF led the health economy analysis section. SC chairs the

666 LEAP. PM, AM, and LJ contributed to the study protocol development. All authors provided

667 intellectual input to the paper and approved its final version.

668

669 Acknowledgements

670 Thank you to all the researchers at the Unit for Social and Community Psychiatry who gave feedback

671 on an early draft of this article, and thanks to all the members of the TACK LEAP whose contributions

672 have improved all aspects of the trial.

673

674 REFERENCES

675 1. King's Fund. Transforming our health care system. Ten priorities for commissioners. The King's

676 Fund. 2015.

677 https://www.kingsfund.org.uk/sites/default/files/field/field_publication_file/10PrioritiesFinal2.pdf. 678 Accessed 19 Jan 2022. 
679 2. Santomauro SF, Mantilla Herrera, AM, Shadid, J, Zheng P, Ashbaugh C, Pigott DM, et al. Global

680 prevalence and burden of depressive and anxiety disorders in 204 countries and territories in 2020

681 due to the COVID-19 pandemic. 2021. 1700:1712.

682

683 3. Jaffe, DH, Rive B, \& Denee TR. The humanistic and economic burden of treatment-resistant

684 depression in Europe: a cross-sectional study. BMC Psychiatry. 2019;

685 https://doi.org/10.1186/s12888-019-2222-4.

686

687 4. Patel V, Chisholm D, Parikh R, Charlson, FJ, Degenhardt, L. Dua, T, et al. Addressing the burden of 688 mental, neurological, and substance use disorders: key messages from Disease Control Priorities, 3rd 689 edition. The Lancet 2016; 1672-85.

690 5. McCrone P, Dhanasiri S, Patel A, Knapp M, Lawton-Smith S. Paying the Price. The cost of mental 691 health care in England to 2026. The King's Fund, 2008.

692 https://www.kingsfund.org.uk/sites/default/files/Paying-the-Price-the-cost-of-mental-health-care-

693 England-2026-McCrone-Dhanasiri-Patel-Knapp-Lawton-Smith-Kings-Fund-May-2008_0.pdf. Accessed $694 \quad 19$ Jan 2022.

6. Jobst A, Brakemeier EL, Buchheim A, Caspar F, Cuijpers P, Ebmeier KP, et al. European Psychiatric

696 Association Guidance on psychotherapy in chronic depression across Europe. Eur Psychiatry. 2016;

697 https://doi.org/10.1016/i.eurpsy.2015.12.003

698 7. Visentini C, Cassidy M, Bird VJ, Priebe S. Social networks of patients with chronic depression: A

699 systematic review. J Affect Disord. 2018; 571-578. doi: 10.1016/j.jad.2018.08.022.

700

701 8. Torpey DC, Klein DN. Chronic depression: update on classification and treatment. Curr Psychiatry Rep. 2008; doi: 10.1007/s11920-008-0074-6. 
704 9. Fonagy P, Rost F, Carlyle JA, McPherson, S, Thomas, R, Pasco Fearon, RM, et alet al. Pragmatic randomized controlled trial of long-term psychoanalytic psychotherapy for treatment-resistant depression: the Tavistock Adult Depression Study (TADS). World Psychiatry. 2015; 312-321. doi:10.1002/wps.20267

708

10. Jaffe DH, Rive B \& Denee TR. The humanistic and economic burden of treatment-resistant depression in Europe: a cross-sectional study. BMC Psychiatry. 2019; 247.

713 11. Angst J, Gamma A, Rössler W, Ajdacic V, Klein DN. Long-term depression versus episodic major 714 depression: Results from the prospective Zurich study of a community sample. Journal of Affective

715 Disorders. 2009; 112-121. https://doi.org/10.1016/j.jad.2008.09.023.

12. Kocsis JH, Gelenberg AJ, Rothbaum B, Klein DN, Trivedi MH, Manber R, et al. Chronic forms of major depression are still undertreated in the 21st century: systematic assessment of 801 patients presenting for treatment. J Affect Disord. 2008; 55-61.

720

13. Simpson A, Hannigan B, Coffey M, et al. Cross-national comparative mixed-methods case study of recovery-focused mental health care planning and co-ordination: Collaborative Care Planning Project (COCAPP). NIHR Journals Library. 2016; https://www.ncbi.nlm.nih.gov/books/NBK343672/ doi: 10.3310/hsdr04050. Accessed 19 Jan 2022. 14. McIntyre RS, Filteau MJ, Martin L, Patry S, Carvalho A, Cha DS, Barakat M, Miguelez M.

727 Treatment-resistant depression: definitions, review of the evidence, and algorithmic approach. J 728 Affect Disord. 2014; 1-7. doi: 10.1016/j.jad.2013.10.043. 
730 15. Priebe S, Kelley L, Omer S, Golden E, Walsh S, Khanom H, et al. The effectiveness of a patient-

731 centred assessment with a solution-focused approach (DIALOG+) for patients with psychosis: A

732 pragmatic cluster-randomised controlled trial in community care. Psychotherapy and

733 Psychosomatics. 2015; 304-303.

734

735

16. Priebe S, Golden E, Kingdon D, Omer S, Walsh S, Katevas K, et al. Effective patient-clinician

interaction to improve treatment outcomes for patients with psychosis: a mixed methods design

(DIALOG+). Health Technology Assessment Programme Grants Appl Res. 2017; 5:6.

738

17. Giacco D, Palumbo C, Strappelli N, Catapano F, Priebe S. Social contacts and loneliness in people

740 with psychotic and mood disorders. Comprehensive Psychiatry. 2016; 59-66.

$741 \quad$ https://doi.org/10.1016/i.comppsych.2015.12.008.

742

18. Slatina Murga S, Janković S, Muhić M, Sikira H, Burn E, Priebe S. Effectiveness of a structured

intervention to make routine clinical meetings therapeutically effective (DIALOG+) for patients with

depressive and anxiety disorders in Bosnia and Herzegovina: A cluster randomised controlled trial.

Psychiatry Research Communications, 2021. https://doi.org/10.1016/j.psycom.2021.100010.

19. Priebe S, Fung C, Sajun, SZ et al. Resource-oriented interventions for patients with severe mental

749 illnesses in low- and middle-income countries: trials in Bosnia-Herzegovina, Colombia and

750 Uganda. BMC Psychiatry. 2019. https://doi.org/10.1186/s12888-019-2148-x

751

752 20. Matanov A, McNamee P, Akther, S. et al. Acceptability of a technology-supported and solution-

753 focused intervention (DIALOG+) for chronic depression: views of service users and clinicians. BMC

Psychiatry, 2021. https://doi.org/10.1186/s12888-021-03256-5 
756 21. Chan AW, Tetzlaff JM, Altman DG, Laupacis A, Gøtzsche PC, Krleža-Jerić K, et al. SPIRIT 2013

757 statement: defining standard protocol items for clinical trials. Ann Intern Med. 2013; 200-7. doi: 10.7326/0003-4819-158-3-201302050-00583.

759

760

22. Priebe S, Huxley P, Knight S, Evans S. Application and results of the Manchester Short Assessment

761 of Quality of Life (MANSA). Int J Soc Psychiatry. 1999; 7-12. doi: 10.1177/002076409904500102.

762

763

23. Montgomery SA \& Åsberg M. A new depression scale designed to be sensitive to change. British

764 Journal of Psychiatry, 1979; 382-389.

765

766

24. Priebe S, McCabe R, Bullenkamp J, Hansson L, Lauber C, Martinez-Leal R, et al. $\underline{\text { Structured }}$

patient-clinician communication and 1-year outcome in community mental healthcare. Cluster

randomised controlled trial (DIALOG). British Journal of Psychiatry, 2007; 420-426.

769

770

25. DIALOG+ Website. www.elft.nhs.uk/tack (2022). Accessed 19 Jan 2022.

771

772

26. Beck AT, Steer RA, \& Brown G. Beck Depression Inventory-II [Database record]. APA PsycTests.

773 1996. https://doi.org/10.1037/t00742-000.

774

775

27. Larsen DL, Attkisson CC, Hargreaves WA, Nguyen TD. Assessment of client/patient satisfaction:

776 development of a general scale. Eval Program Plann. 1979; 197-207. doi: 10.1016/01497189(79)90094-6.

778

779 28. Guy W. ECDEU Assessment Manual for Psychopharmacology. Rockville, MD: U.S. Department of 780 Health, Education, and Welfare. 1976. 
782 29. Al-Janabi H, Flynn TN, Coast J. Development of a self-report measure of capability wellbeing for

783 adults: the ICECAP-A. Qual Life Res. 2012; 167-76. doi: 10.1007/s11136-011-9927-2.

784

785

30. Herdman M, Gudex C, Lloyd A, Janssen M, Kind P, Parkin D, et al. Development and preliminary

786

testing of the new five-level version of EQ-5D (EQ-5D-5L). Qual Life Res. 2011; 1727-36. doi:

787

10.1007/s11136-011-9903-x.

788

31. Beecham J, \& Knapp Martin. Costing psychiatric interventions. In: Thornicroft G, editors.

789

Measuring Mental Health Needs. London: Gaskell; 2001. p. 200-224.

790

32. Van Hout B, Al M, Gordon G, Rutten F. Costs, effects and c/e-ratios alongside a clinical trial.

791

Health Economics. 1994; 309-319.

792

793 33. NICE. National Institute for Health and Care Excellence Guide to the methods of technology

794 appraisal. National Institute for Health and Care Excellence. 2013.

795 https://www.nice.org.uk/process/pmg9/resources/guide-to-the-methods-of-technology-appraisal-

796

2013-pdf-2007975843781. Accessed 19 th Jan 2022.

797

798

34. Black W: The CE plane: A graphic representation of cost-effectiveness. Medical Decision Making.

799 1990; 212-214.

800

801

35. Flynn TN, Huynh E, Peters TJ, Al-Janabi H, Clemens S, Moody A, Coast J. Scoring the ICECAP-A

802 capability instrument. Estimation of a UK general population tariff. Health Econ. 2015; 258-69.

803

804 36. TACK website. https://www.elft.nhs.uk/tack. Accessed on 19 th Jan 2022.

805 
